\title{
Improving the Starting Characteristics of Single-Phase Induction Motors with an Auxiliary-Winding Current Control
}

\author{
Satoshi Isaka* Student Member, Toshiya Yoshida ${ }^{* a)}$ Senior Member
}

(Manuscript received Feb. 25, 2019, revised June 17, 2019)

\begin{abstract}
This paper proposes a novel starting method for single-phase induction motors. Induction motors are unable to obtain sufficient starting torque with only a single-phase source. Therefore, the proposed method increases the starting torque by controlling the auxiliary-winding current with a triode for alternating current (TRIAC) and a very small microcontroller. The auxiliary winding is connected to the main windings via the TRIAC and a small capacitor. The intermittent TRIAC connections provide a pulse current series, i.e., half-cycle waveforms of the resonant current. The fundamental component of this current is sufficiently large and leads the main winding current; hence, a better two-phase current is realized. Thus, the proposed control achieves a large starting torque by utilizing only a small capacitor; it does not use any electrolytic capacitors. The effectiveness of the control is verified by theoretical and experimental methods. The proposed method obtained almost twice the starting torque of the conventional method, using the same capacitor.
\end{abstract}

Keywords: single-phase induction motor, TRIAC, resonant current, starting torque

\section{Introduction}

Single-phase induction motors are widely used in industrial machinery and home appliances, due to their high reliability and low cost. Since the induction motor is unable to obtain sufficient starting torque with only a single-phase source, a mechanism for generating a rotating magnetic field must be employed.

Various methods are used for starting single-phase motors. When a large starting torque is expected, the capacitor starting method of which the auxiliary winding is connected to the source via a capacitor may be used. In this method, a large, inexpensive AC electrolytic capacitor is popular. However, when an electrolytic capacitor is placed on a single-phase induction motor, its lifetime is shortened because of the heat of the motor ${ }^{(1)}$. A large capacitor should be used for starting, while a small capacitor should be used for running.

A centrifugal governor that switches to a small-capacity capacitor is necessary to prevent motor-efficiency degradation in the steady state; this mechanical moving part, however, may cause low reliability. Thus, starting methods without centrifugal governors ${ }^{(2)}$ or electrolytic capacitors ${ }^{(3)}$ have been proposed. An improved method using an inverter has also been proposed ${ }^{(4)}$; unfortunately, utilizing the inverter increases the cost.

This paper proposes a novel method for starting singlephase induction motors. The auxiliary-winding current is controlled with a simple circuit that equips a TRIAC (triode for alternating current) and a very small microcontroller. Using the proposed method, a large starting torque can be obtained with a small capacitor, e.g., a film capacitor, which

a) Correspondence to: Toshiya Yoshida. E-mail: tyoshida@eee. dendai.ac.jp

* Tokyo Denki University

5, Senjyu-Asahicho, Adachi-ku, Tokyo 120-8551, Japan can also be used during steady-state motor operation. The TRIAC controls both the function of the auxiliary winding current in the starting operation, and also the centrifugal governor, which switches the motor operation to a steady state; no mechanical moving parts are used.

In this paper, we first derive the starting torque, based on an equivalent circuit of the induction motor. Next, we show the validity of the proposed method by experimentation. The starting characteristic of the proposed method is compared with a conventional method (capacitor motor) in which a capacitor is permanently installed between the AC power source and the auxiliary winding. Then, the current distortion and power-source impedance are discussed, and conclusions are drawn.

\section{Proposed Starting Method}

\subsection{Proposed Starting Method with TRIAC}

Figure 1 shows the structure of the proposed starting method. The auxiliary winding is connected to the power source via the TRIAC and the capacitor. The TRIAC is triggered after a fixed delay starting from the zero-cross point of the source voltage, as shown in Fig. 2. Then, a resonant

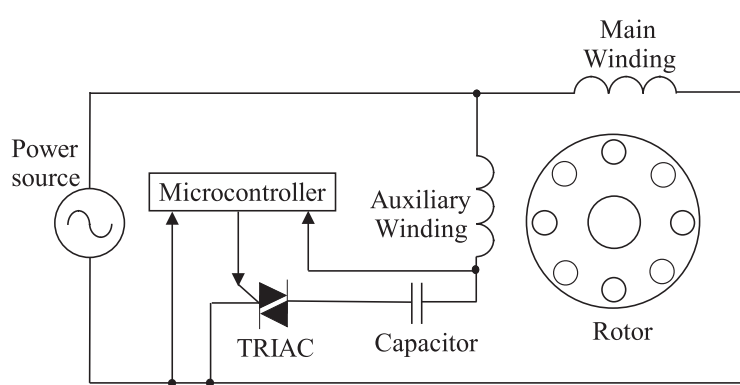

Fig. 1. Proposed circuit to increase the torque of a single-phase induction motor 


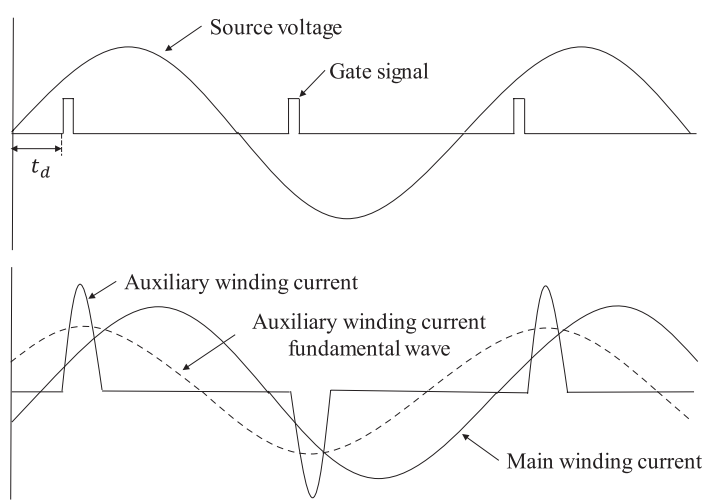

Fig. 2. Source voltage and winding current

current flows through the auxiliary winding due to the inductance of the auxiliary winding and the capacitor; the halfcycle wave of the resonant current is obtained after the trigger.

When the resonant frequency is much higher than the source frequency, and the trigger is given in each half-cycle of the source voltage, successive quasi-impulse currents are observed; the auxiliary winding current is a periodic quasiimpulse series. The fundamental component of this current is a sinusoidal wave, of which the peak is near the triggered point.

If the delay time is shorter than the quarter-cycle period of the source voltage, the fundamental current of the auxiliary winding leads the source voltage. On the other hand, the main winding current lags behind the source voltage, due to the inductive impedance of the main winding. Thus, the motor is driven by a quasi-two-phase current. The fundamental component becomes larger than the current of the capacitor connected directly between the source and the winding. As a result, the lack of the auxiliary winding current is improved and the proposed method obtains a larger starting torque.

2.2 TRIAC Gate Signal Control A very small microcontroller (PIC10F322, Microchip Technology, Inc.) is used to control the TRIAC's gate signal. The source voltage is monitored by a built-in A/D converter to detect the zerocross timing of the source voltage. The auxiliary winding voltage is also measured as the EMF (electromotive force), which gives the rotor-speed information used for the centrifugal governor function.

If the firing angle (delay time) of the TRIAC is set close to $90^{\circ}$, the auxiliary-winding current increases because the resonant circuit, consisting of the auxiliary winding and the capacitor, is energized by the peak voltage of the source. In this case, the fundamental component of the auxiliary-winding current is almost in phase with the source voltage; the leading current cannot be obtained. On the other hand, if the firing angle is set at about $0^{\circ}$, the lead angle with respect to the voltage is about $90^{\circ}$, though the fundamental current decreases. Consequently, the optimum firing angle should be between $0^{\circ}$ and $90^{\circ}$.

\section{Calculation of the Starting Torque with an Equivalent Circuit}

In this section, the starting torque with respect to the firing angle is theoretically calculated, based on an equivalent

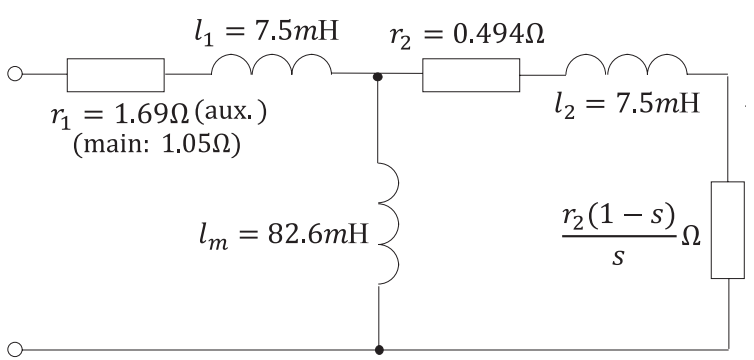

Fig. 3. Equivalent circuit of a single-phase induction motor used to calculate the starting torque

circuit of the induction motor. The starting torque of the proposed method is calculated from the fundamental voltages and currents of the main and auxiliary windings. Figure 3 shows the equivalent circuit of the single-phase induction motor. The physical angle between the main and auxiliary windings is $\pi / 2$. The constants of the equivalent circuit were identified by an actual motor test with a balanced two-phase voltage source. The motor can be assumed as a balanced two-phase motor because the effective turn ratio of the main winding and the auxiliary winding $\left(a=N_{a} / N_{m}\right)$ is 1.0. Although only the primary resistances of the main and auxiliary windings are slightly different $\left(r_{1}\right.$ of main is $1.05 \Omega$ and auxiliary1.69 $\Omega$ ), this difference hardly affects the calculated starting torque. In the following discussions, $r_{1}$ of the auxiliary winding is applied.

3.1 Resonant Frequency First, the resonant frequency of the resonant circuit (the auxiliary winding and the capacitor) is calculated, using the equivalent circuit in the start-up condition: slip $\mathrm{s} \rightarrow 1$.

In the equivalent circuit of Fig. $3, r_{2}$ is the secondary resistance, $l_{1}$ is the primary leakage inductance, $l_{2}$ is the secondary leakage inductance, and $l_{m}$ is the excitation inductance. $C$ is the capacitance of the starting capacitor, and $\omega_{\text {res }}$ is the resonant angular frequency. The auxiliary winding parameters of the primary side are converted with effective turn ratio $a$ as follows ${ }^{(6)}: V_{s}^{\prime}=V_{s} / a, C^{\prime}=a^{2} C, l_{1}^{\prime}=l_{1} / a^{2}$, and $r_{1}^{\prime}=r_{1} / a^{2}$, where $V_{s}$ is the amplitude of the source voltage. The experimental motor of this paper, however, does not require these conversions: $a=1.0$. When the resonant circuit resonates, the circuit equation is given as

$$
\begin{aligned}
& \left\{l_{1}^{\prime}\left(l_{2}+l_{m}\right)^{2}+l_{2} \cdot l_{m}\left(l_{2}+l_{m}\right)\right\} \omega_{\text {res }}{ }^{4} \\
& \quad+\left\{l_{1}^{\prime} \cdot r_{2}^{2}-\frac{\left(l_{2}+l_{m}\right)^{2}}{C^{\prime}}+l_{m} \cdot r_{2}{ }^{2}\right\} \omega_{\text {res }}{ }^{2}-\frac{r_{2}^{2}}{C^{\prime}}=0 .
\end{aligned}
$$

The angular frequency $\omega_{\text {res }}$ is

$$
\omega_{\text {res }}=\sqrt{\frac{-y+\sqrt{y^{2}-4 x z}}{2 x}},
$$

where $x$ is the coefficient of $\omega_{\text {res }}^{4}, y$ is that of $\omega_{\text {res }}^{2}$, and $z$ is the constant term of Eq. (1).

3.2 Peak Value of the Resonant Current The peak value of the resonant current $I_{\text {apeak }}$ is calculated. Figure 4 shows the source voltage and the auxiliary-winding current's waveforms for the proposed method. If the resonant current is regarded as a half-cycle pulse sinusoidal, the pulse width 


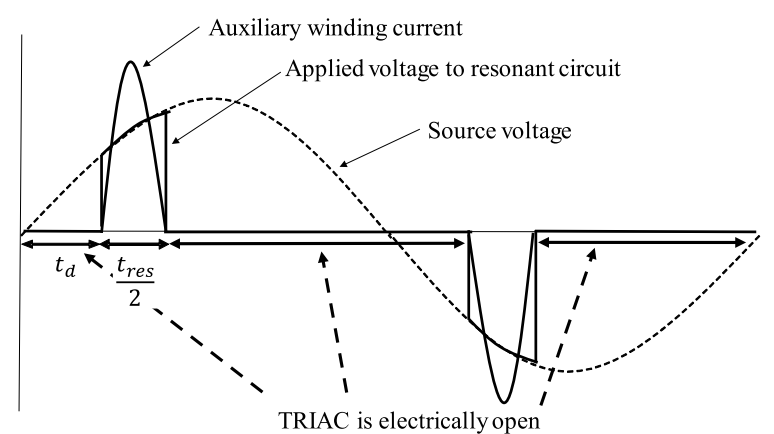

Fig. 4. Voltage and current waveforms of auxiliary winding

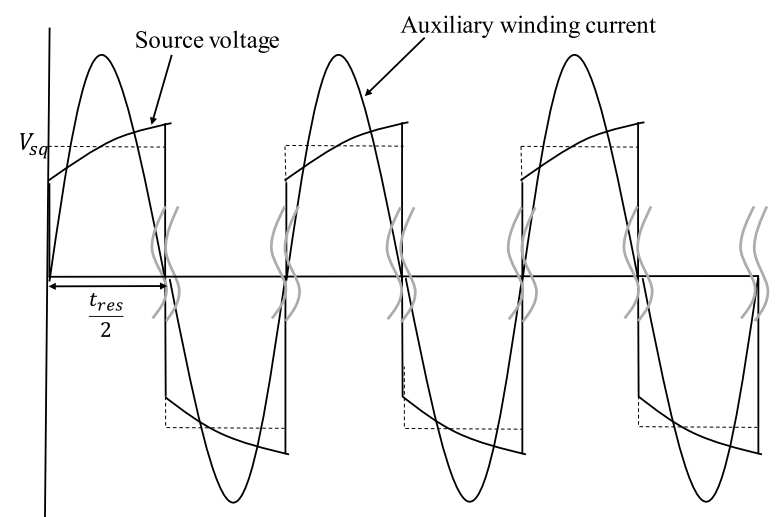

Fig. 5. Source voltage and auxiliary winding current assumed to be continuous

is assumed to be half of the resonant period, $t_{\text {res }}=\pi / \omega_{\text {res }}$. Since the resonant period is chosen to be much smaller than the source voltage period, the voltage can be assumed to be constant in the pulse-width duration.

Now, consider a waveform reconstructed so that the zerocurrent sections are removed from the auxiliary current waveform, as shown in Fig. 5. The reconstructed current waveform of Fig. 5 is sinusoidal and results from the resonant circuit powered by the square-wave voltage.

Let the peak voltage of the imaginary square wave be

$$
V_{s q}=V_{s}^{\prime} \cdot \sin \left(\omega_{s}\left(t_{d}+\frac{t_{r e s}}{4}\right)\right)
$$

where $\omega_{s}$ is the angular frequency of the source, and $t_{d}$ is the delay time. Thus, the peak value of the resonant current is given by

$$
I_{\text {apeak }}=\frac{4}{\pi} \cdot \frac{V_{s q}}{r_{\text {res }}},
$$

where $r_{\text {res }}$ is the impedance (resistance) when the resonant circuit resonates. The harmonic voltage of the square wave is ignored. $r_{r e s}$ is given as follows:

$$
r_{\text {res }}=r_{1}^{\prime}+\frac{\omega_{\text {res }}^{2} \cdot l_{m}^{2} \cdot r_{2}}{r_{2}^{2}+\omega_{r e s}^{2}\left(l_{2}+l_{m}\right)^{2}} .
$$

3.3 Calculation of the Starting Torque The amplitude of the fundamental component of the resonant current through the auxiliary winding is given as

$$
A_{a 1}=\frac{2}{T_{s}} \int_{-\frac{T_{s}}{2}}^{\frac{T_{s}}{2}} i_{a}(t) \cdot \cos \left(\omega_{s} \cdot t\right) d t
$$

$$
=\frac{4 \omega_{s}}{\pi} \int_{0}^{\frac{\text { tres }}{4}} I_{\text {apeak }} \cdot \cos \left(\omega_{\text {res }} \cdot t\right) \cdot \cos \left(\omega_{s} \cdot t\right) d t \cdots
$$

where $T_{s}$ is the period of the power source $\left(2 \pi / \omega_{s}\right)$, and $i_{a}$ is the auxiliary winding current. The phase difference between the fundamental wave of the auxiliary-winding current and the source voltage is given by

$$
\theta_{i a}=\frac{\pi}{2}-\omega_{s}\left(t_{d}+\frac{t_{r e s}}{4}\right)
$$

The resistance $r$ and the reactance $x_{l}$ of the main winding at the source angular frequency are given as

$$
\begin{aligned}
& r=r_{1}+\frac{\omega_{s}^{2} \cdot l_{m}^{2} \cdot r_{2}}{r_{2}{ }^{2}+\omega_{s}^{2}\left(l_{2}+l_{m}\right)^{2}}, \ldots \ldots \ldots \ldots \ldots \ldots \ldots \\
& x_{l}=\omega_{s} \cdot l_{1}+\frac{\omega_{s}^{3} \cdot l_{m} \cdot l_{2}\left(l_{2}+l_{m}\right)+\omega_{s} \cdot l_{m} \cdot r_{2}{ }^{2}}{r_{2}{ }^{2}+\omega_{s}{ }^{2}\left(l_{2}+l_{m}\right)^{2}} .
\end{aligned}
$$

The amplitude $A_{m}$ and the phase $\theta_{i m}$ of the main-winding current are given as

$$
\begin{aligned}
A_{m} & =\frac{V_{s}}{\sqrt{r^{2}+x_{l}^{2}}}, \\
\theta_{i m} & =\tan ^{-1}\left(\frac{x_{l}}{r}\right) .
\end{aligned}
$$

Using equations (6), (7), (10), and (11), the main-winding current $\dot{I}_{m}$ and the fundamental wave of the auxiliary-winding current $\dot{I}_{a 1}$ are given in the following.

$$
\begin{aligned}
& \dot{I}_{m}=A_{m}\left(\cos \theta_{i m}+j \sin \theta_{i m}\right), \\
& \dot{I}_{a 1}=A_{a 1}\left(\cos \theta_{i a}-j \sin \theta_{i a}\right) .
\end{aligned}
$$

The fundamental waves of the main-winding current and the auxiliary-winding current are unbalanced. For this reason, the torque is calculated by separating these currents into positive and negative phases. The positive-phase current of the main winding $\dot{I}_{m p}$, the positive-phase current of the auxiliary winding $\dot{I}_{a p}$, the negative-phase current of the main winding $\dot{I}_{m n}$, and the negative-phase current of the auxiliary winding $\dot{I}_{a n}$ are

$$
\begin{aligned}
& \dot{I}_{m p}=\frac{1}{2}\left(\dot{I}_{m}-j \dot{I}_{a 1}\right), \cdot \\
& \dot{I}_{a p}=j \frac{1}{2}\left(\dot{I}_{m}-j \dot{I}_{a 1}\right), \\
& \dot{I}_{m n}=\frac{1}{2}\left(\dot{I}_{m}+j \dot{I}_{a 1}\right), \cdot \\
& \dot{I}_{a n}=-j \frac{1}{2}\left(\dot{I}_{m}+j \dot{I}_{a 1}\right),
\end{aligned}
$$

respectively. Then, these currents are divided into an excitation current and a secondary current, as follows:

$$
\begin{aligned}
& \dot{I}_{2 m p}=\dot{I}_{m p} \cdot \frac{j \omega_{s} \cdot l_{m}}{r_{2}+j \omega_{s}\left(l_{2}+l_{m}\right)}, \\
& \dot{I}_{1 a p}=\dot{I}_{a p} \cdot \frac{r_{2}+j \omega_{s} \cdot l_{2}}{r_{2}+j \omega_{s}\left(l_{2}+l_{m}\right)}, \\
& \dot{I}_{2 m n}=\dot{I}_{m n} \cdot \frac{j \omega_{s} \cdot l_{m}}{r_{2}+j \omega_{s}\left(l_{2}+l_{m}\right)}, \\
& \dot{I}_{1 a n}=\dot{I}_{a n} \cdot \frac{r_{2}+j \omega_{s} \cdot l_{2}}{r_{2}+j \omega_{s}\left(l_{2}+l_{m}\right)} \cdots
\end{aligned}
$$




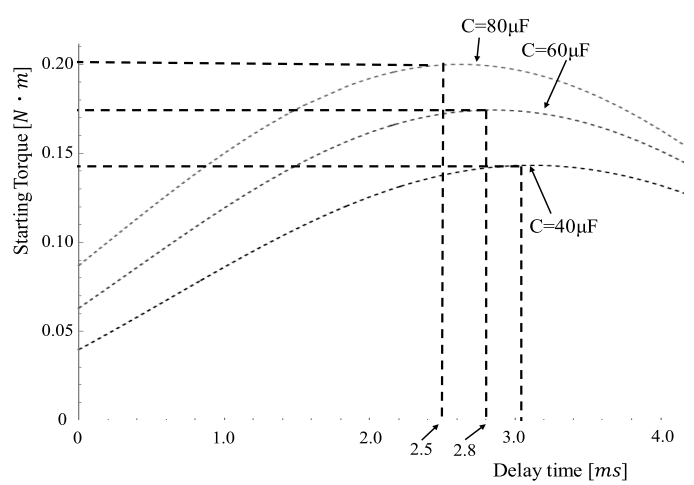

Fig. 6. Calculated starting torque vs. delay time with various capacitance values

Here, $\dot{I}_{2 m p}$ is the positive-phase secondary current of the main winding, $\dot{I}_{1 a p}$ is the positive-phase excitation current of the auxiliary winding, $\dot{I}_{2 m n}$ is the negative-phase secondary current of the main winding, and $\dot{I}_{1 a n}$ is the negative-phase excitation current of the auxiliary winding.

Therefore, the positive-phase torque and the negativephase torque are calculated. The starting torque of the proposed method is given by

$$
\tau=\frac{P}{2} l_{m}\left\{\operatorname{Re}\left[\dot{I}_{2 m p} \cdot \overline{\dot{I}_{1 a p}}\right]+\operatorname{Re}\left[\dot{I}_{2 m n} \cdot \overline{\dot{I}_{1 a n}}\right]\right\}, \cdots
$$

where $P$ is the number of poles.

Figure 6 shows the starting torque using (22) with respect to the delay time with various capacitances of the capacitor. The delay time at which the starting torque becomes the maximum depends on the capacitance. As the capacitance increases, the optimum delay time decreases. Hence, the delay time must be chosen according to the capacitance to obtain the maximum starting torque.

\section{Measurement and Investigation by an Actual Motor Test}

The starting torque measured by an actual motor test is compared with the result calculated from the equivalent circuit. A 400-W 50-Hz 100-V 2-pole motor is used. The supply voltage is $40 \mathrm{~V}$ in the experiments. A disk with a much greater moment of inertia $\left(0.01215 \mathrm{Nm}^{2}\right)$ than that of the rotor is attached to the single-phase induction motor. The output torque is measured in the acceleration process with this inertia by a time derivation of the speed; the torque in acceleration can be assumed as static torque. The actual motor tests are performed with various delay times from $0.5 \mathrm{~ms}$ to $4.0 \mathrm{~ms}$. The capacitance is chosen as $40 \mu \mathrm{F}, 60 \mu \mathrm{F}$, and $80 \mu \mathrm{F}$. In this motor, the optimum capacitance for capacitor-run operation (steady-state operation) is $60 \mu \mathrm{F}$.

4.1 Measurement and Investigation of Starting Torque Table 1 shows the optimum delay time and the starting torque at that delay time by experimentation and calculation. The optimum delay time and the starting torque values calculated by the equivalent circuit agreed well with the results of the actual motor test.

Table 2 compares the starting torque of the conventional method and the proposed method in an actual machine test. The delay time was set at the optimum. In the test of the conventional method, the auxiliary winding was connected to the
Table 1. Starting torque with optimum delay time

\begin{tabular}{|r|c|c|c|c|}
\hline \multirow{2}{*}{ Cap. } & \multicolumn{2}{|c|}{ Starting torque $[\mathrm{N} \cdot \mathrm{m}]$} & \multicolumn{2}{c|}{ Optimum delay time[ms] } \\
\cline { 2 - 5 } & Experiment & Calculated & Experiment & Calculated \\
\hline $40 \mu \mathrm{F}$ & 0.150 & 0.143 & 3.0 & 3.11 \\
\hline $60 \mu \mathrm{F}$ & 0.175 & 0.174 & 2.8 & 2.85 \\
\hline $80 \mu \mathrm{F}$ & 0.205 & 0.200 & 2.5 & 2.62 \\
\hline
\end{tabular}

Table 2. Starting torque in actual machine test

\begin{tabular}{|r|c|c|}
\hline Cap. & Conventional method $[\mathrm{N} \cdot \mathrm{m}]$ & Proposed method $[\mathrm{N} \cdot \mathrm{m}]$ \\
\hline $40 \mu \mathrm{F}$ & 0.050 & 0.150 \\
\hline $60 \mu \mathrm{F}$ & 0.075 & 0.175 \\
\hline $80 \mu \mathrm{F}$ & 0.110 & 0.205 \\
\hline
\end{tabular}

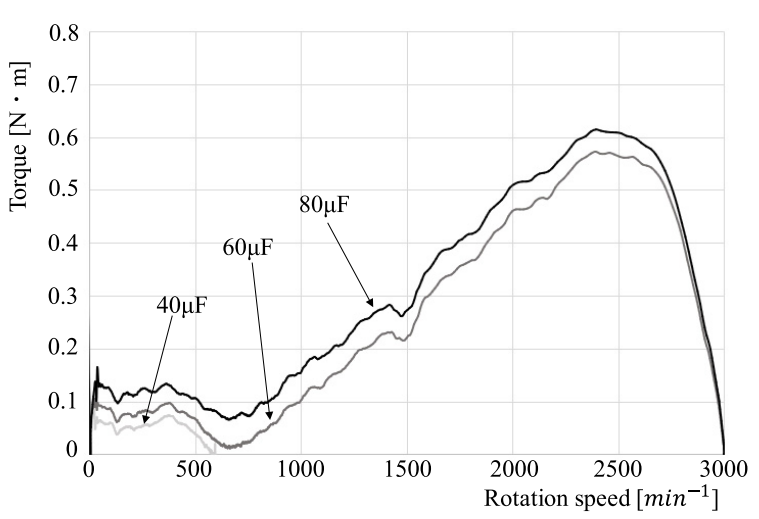

(a) Conventional method

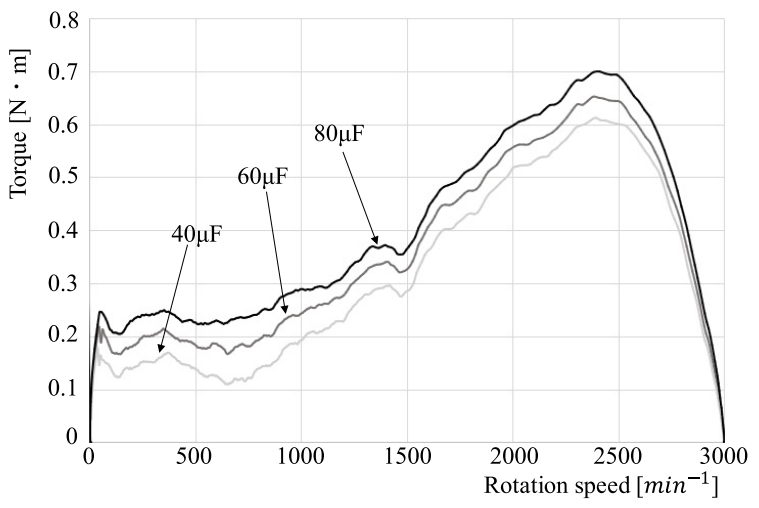

(b) Proposed method

Fig. 7. Experimental torque characteristics, illustrating the higher torque of the proposed model

source via the capacitor without the TRIAC. The proposed method obtained two or three times more starting torque than the conventional method.

\subsection{Torque Characteristics in Acceleration Process}

Figure 7 shows the experimental torque-speed characteristics. In Fig. 7(a), which shows the conventional method, a drop in torque is found at around $600 \mathrm{~min}^{-1}$, due to the "crawling" effect caused by the special harmonics of the rotor. This effect appears with unbalanced main and auxiliary winding voltages. Thus, the speed did not reach the nominal speed when $40 \mu \mathrm{F}$ was applied.

On the other hand, in the actual motor test with the proposed method shown in Fig. 7(b), the rotor was accelerated to the nominal speed, even though a small capacitance was utilized. The torque of the proposed method in the lowerspeed region is remarkably increased and the torque drop 


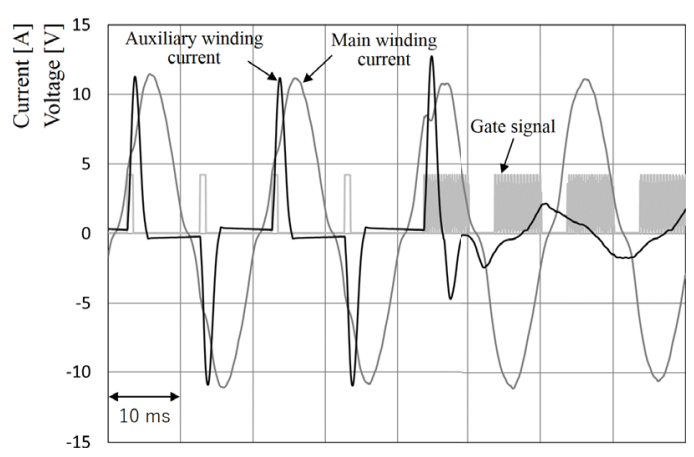

Fig. 8. Winding current and gate signal when gate pattern is changed

becomes inconspicuous. The lower-speed torque of the proposed method with $40 \mu \mathrm{F}$ is considerably greater than that of the conventional with $80 \mu \mathrm{F}$. Consequently, the capacitance can be reduced to half or less, as compared to the conventional method. The proposed method achieved startingtorque boost with the optimum capacitance $(60 \mu \mathrm{F})$ for the capacitor-run operation. Thus, the starting torque of conventional capacitor motors may be improved by this method.

The capacitor peak voltage of $40 \mu \mathrm{F}$ was $165 \mathrm{~V}_{0-\mathrm{p}}$ : about 2.9 times of the peak source voltage. A capacitor rated voltage has to be chosen as higher due to the resonant phenomenon. A film capacitor with little higher rated voltage may not be an obstacle in cost.

4.3 Switching to a Motor Capacitor In the proposed method, the capacitor can be utilized not only in the starting operation but also in the steady-state operation. The motor works as a "capacitor motor" by a continuous electrical connection of the TRIAC. The capacitance is chosen to improve the steady-state efficiency of the motor. The starting torque with the proposed method is enough to start a reasonable load without an additional starting (large) capacitor: only with a small capacitor for capacitor-run operation.

Figure 8 shows the waveforms when the trigger-pulse pattern is changed at around the nominal speed. Gate pulses synchronous with the source voltage with an optimum delay are applied in the acceleration, and then successive frequent pulses are done in the steady state. The auxiliary winding current also changes from resonant pulses to a sinusoidal. The current transition is smooth; the transient is observed for only about half of a source cycle.

The switch to motor-capacitor mode is performed by monitoring the auxiliary-winding voltage. The EMF is observed in the terminal voltage within the duration in which the resonant pulses disappear.

\section{Current Distortion}

In the proposed method, the source current is a mixture of the sinusoidal current of the main winding and the quasiimpulse current of the auxiliary winding. Figure 9 shows the harmonic distortion ratio of the source current with various capacitances. These were calculated with the parameters of Fig. 3 using the PSIM simulator (Myway Plus Corporation). The rotor is locked. The harmonic distortion lies at around $16 \%$, though the distortion is slightly smaller when a smaller capacitor is used. The distortion is not extremely

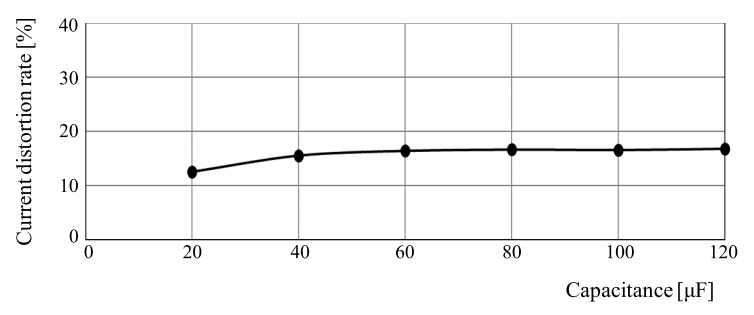

Fig. 9. Current distortion ratio (THD) with respect to capacitance

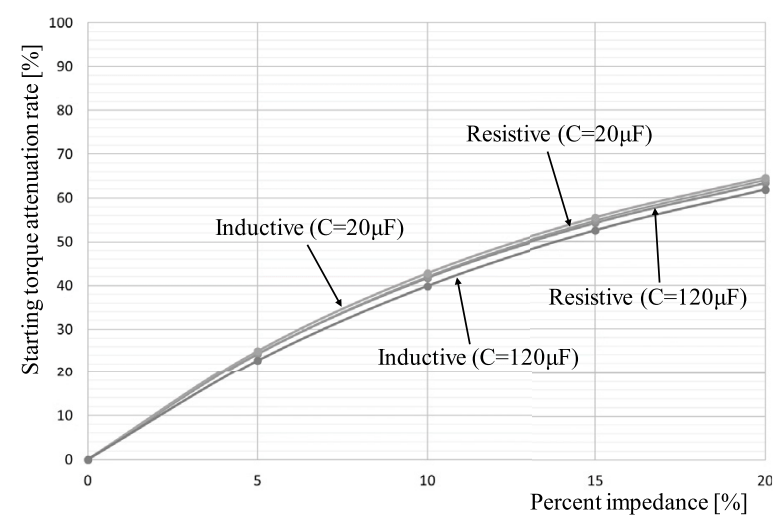

(a) Conventional starting method

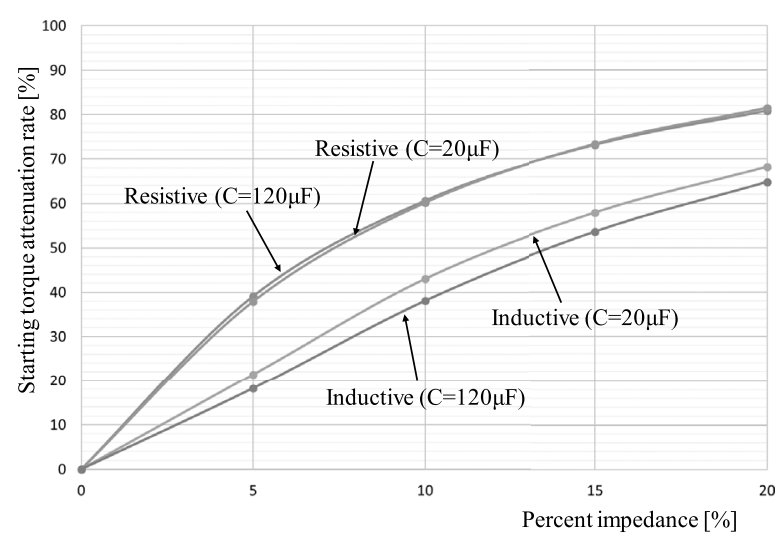

(b) Proposed method

Fig. 10. Starting torque degradation ratio with various percent source impedances

large because the main-winding current is a large sinusoidal while starting, as shown in Fig. 8.

The harmonic current occurs only when the system is in start-up transient mode. A typical load may be accelerated to the nominal speed within a few seconds or less. The proposed method gives practically no harmonics problems. In the Japanese Guidelines for Reduction of Harmonic Emissions, harmonic currents lasting for not more than 10 seconds when a piece of equipment is moved into or out of operation, are disregarded ${ }^{(5)}$.

\section{Influence by Power-Source Impedance}

Since the resonant current of the auxiliary winding is negatively affected by the source impedance, Fig. 10 shows the starting-torque degradation ratio with respect to the source impedance. The torque is normalized with that obtained by an ideal voltage source. The impedance is also normalized with the motor-winding impedance at the rated operation and is considered to be resistive or inductive. 
In the conventional method, the starting torque decreases as the impedance increases, regardless of the capacitance and the impedance characteristics. In the proposed method, when the source has resistive impedance, the starting torque considerably decreases; the resonant current is damped and the fundamental current of the auxiliary winding shrinks. On the other hand, the degradation of the proposed method with inductive impedance is almost the same as the conventional. In practical use, the problem does not become obvious because the source impedance is almost inductive.

\section{Conclusions}

This paper proposed a novel starting method for a singlephase induction motor. The proposed method is cost effective and obtained a large starting torque with a small-capacitance capacitor. The calculated starting torque with an equivalent circuit agreed well with the result of the experiments.

In the proposed method, the starting torque was twice or greater than that of the conventional method with the same capacitor. Therefore, a small-capacitance capacitor could be employed, making long-life or film capacitors possible. Moreover, the single capacitor not only works as a starting capacitor but also as a motor capacitor. Switching from startup to steady-state operation is accomplished by changing the gate signal according to the auxiliary-winding voltage, without centrifugal force switches.

The method was verified with a balanced motor (turn ratio $=1.0)$. Further studies on unbalanced motors $(\gg 1.0)$ are needed.

\section{Acknowledgment}

This research has been supported by Ebara Corporation.

\section{References}

( 1 ) M.L. Gasperi: "A Method for Predicting the Expected Life of Bus Capacitors", IEEE-IAS Annual Meeting, Vol.3, pp.1042-1047 (1997)
( 2 ) T. Takaku, J. Narushima, T. Isobe, T. Kitahara, and R. Shimada: "Power Factor Correction of Single-Phase Induction Motor Using Magnetic Energy Recovery Switch", IEEJ D, Vol.126, No.9, pp.1214-1219 (2006)

( 3 ) R. Darbali-Zamora, D.A. Merced-Cirino, A.J. Diaz-Castillo, and E.I Ortiz-Rivera: "Single-Phase Induction Motor Alternate Start-up and Speed Control Method for Renewable Energy Applications", IEEE-ICRERA 2014, pp.743-748 (2014)

( 4 ) J.R. Wells, B.M. Nee, M. Amrhein, P.T. Krein, and P.L. Chapman: "LowCost Single-Phase Powered Induction Machine Drive for Residential Application", 19th Annual IEEE Applied Power Electronics Conference and Exposition 2004, Vol.3, No.1, pp.1579-1583 (2004)

( 5 ) E. Sakashita, K. Yukihira, Y. Ogihara, N. Fujii, K. Hasegawa, F. Kamiya, A. Tanaka, and M. Yoshida: "Japanese Guideline for Reduction of Harmonic Emission", IEC SC77A Japanese National Committee (2001)

( 6 ) M. Suzuki, M. Murase, H. Igarashi, M. Kodama, and Y. Ito: "Programming for Calculation of Characteristic of Condenser-Motor at Normal Operation", Aichi Electric Technical Report, No.23, pp.23-29 (2000)

Satoshi Isaka (Student Member) was born 25 May 1993. In 2015,

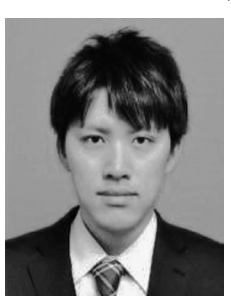
he graduated from Tokyo Denki University (School of Engineering, Department of Electrical and Electronic Engineering). He completed a Master's course at Tokyo Denki University in 2019.

Toshiya Yoshida (Senior Member) was born 21 January 1971. In

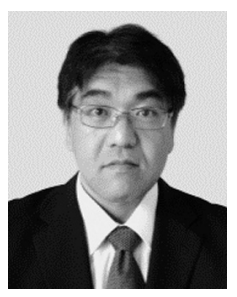
1996, he completed a Master's course at Tokyo Denki University (Graduate School of Science and Technology, Department of Applied Electronics), and was employed by the university as an assistant (Faculty of Science and Technology, Department of Applied Electronics). He completed a doctorate in Engineering in 1999. In 2000, he was an assistant (Electrical Engineering), in 2001, a lecturer, in 2004, an assistant professor, and in 2007, an adjunct professor (Department of Electrical and Electronic Engineering). Since 2012, he has been a full professor. He is a member of the IEEE and EPE. 